\title{
Seletividade de herbicidas residuais e da mistura com glyphosate aplicados após a poda da mandioca 'Fécula Branca''
}

\section{Selectivity of residual herbicides and mixture with glyphosate applied after the pruning of cassava 'Fécula Branca'}

Neumarcio Vilanova Costa ${ }^{2}$; Milciades Melgarejo Arrúa ${ }^{3}$; Daniel Adriano Sontag ${ }^{4}$; Devair Carlos de Andrade $^{5}$; José Barbosa Duarte Júnior ${ }^{6}$

Resumo - As variedades de mandioca (Manihot esculenta L. Crantz) destinadas para a indústria de fecularia geralmente são podadas no período de repouso fisiológico das plantas, sendo necessária a adoção de práticas de manejo das plantas daninhas para evitar a competição no segundo ciclo de desenvolvimento da cultura. Diante do exposto, o objetivo foi avaliar a seletividade de herbicidas pré-emergentes e da mistura destes com glyphosate em aplicações após a poda da mandioca 'Fécula Branca'. O delineamento experimental utilizado foi o de blocos ao acaso, com quatro repetições. Os tratamentos avaliados foram: glyphosate (720 $\left.\mathrm{g} \mathrm{ha}^{-1}\right)$, carfentrazone $\left(25 \mathrm{~g} \mathrm{ha}^{-1}\right)$, chlorimuron-ethyl $\left(10 \mathrm{~g} \mathrm{ha}^{-1}\right)$, clomazone $\left(1.080 \mathrm{~g} \mathrm{ha}^{-1}\right)$, flumioxazin (83 $\left.\mathrm{g} \mathrm{ha}^{-1}\right)$, metribuzin $\left(480 \mathrm{~g} \mathrm{ha}^{-1}\right)$ metsulfuron-methyl $\left(2,4 \mathrm{~g} \mathrm{ha}^{-1}\right)$, glyphosate + carfentrazone $(720+$ $\left.25 \mathrm{~g} \mathrm{ha}^{-1}\right)$, glyphosate + chlorimuron-ethyl $\left(720+10 \mathrm{~g} \mathrm{ha}^{-1}\right)$, glyphosate + clomazone $(720+1.080$ $\left.\mathrm{g} \mathrm{ha}^{-1}\right)$, glyphosate + flumioxazin $\left(720+83 \mathrm{~g} \mathrm{ha}^{-1}\right)$, glyphosate + metribuzin $\left(720+480 \mathrm{~g} \mathrm{ha}^{-1}\right)$, glyphosate + metsulfuron-methyl $\left(720+2,4 \mathrm{~g} \mathrm{ha}^{-1}\right)$, além de uma testemunha sem aplicação. Avaliou-se a porcentagem de fitointoxicação, altura de planta, produtividade de raízes e o teor de fécula da mandioca. Os herbicidas aplicados isolados e em mistura não causaram sintomas de fitointoxicação nas plantas de mandioca após a aplicação. Os herbicidas aplicados isolados e em mistura não reduziram a altura de planta, produtividade de raízes e o teor de fécula da mandioca em relação à testemunha sem aplicação. Conclui-se que a aplicação isolada dos herbicidas residuais e da mistura com glyphosate apresentaram-se seletivos, quando aplicados após a poda das plantas de mandioca 'Fécula Branca'.

Palavras-chaves: Manihot esculenta L. Crantz, mistura em tanque, planta daninha

Abstract - Cassava varieties (Manihot esculenta L. Crantz) intended for industrial starch manufacturers are usually pruned during the period of physiological rest of the plants, requiring the adoption of management practices to prevent weed competition in the second cycle of crop development. The objective was to evaluate the selectivity of pre-emergent herbicides applied

\footnotetext{
${ }^{1}$ Recebido para publicação em 15/01/2014 e aceito em 10/05/2015.

${ }^{2}$ Docente do Centro de Ciência Agrárias da Universidade Estadual do Oeste do Paraná (Unioeste), Rua Pernambuco, C.P. 91, CEP 85960-000, Marechal Cândido Rondon, PR. E-mail: neumarcio.costa@unioeste.br.(Autor para correspondência)

${ }^{3}$ Doutorando do Programa de Pós-graduação em Agronomia da Unioeste - (PPGA)

${ }^{4}$ Discentes do curso de Agronomia da Universidade Estadual do Oeste do Paraná (Unioeste).

${ }^{5}$ Discentes do curso de Agronomia da Universidade Estadual do Oeste do Paraná (Unioeste).

${ }^{6}$ Docente do Centro de Ciência Agrárias da Universidade Estadual do Oeste do Paraná (Unioeste), Rua Pernambuco, CEP 85960-000, Marechal Cândido Rondon, PR.
} 
alone or in tank mix with glyphosate in applications after pruning of cassava 'Fécula Branca'. The experimental design was a randomized block with four replications. The treatments were: glyphosate $\left(720 \mathrm{~g} \mathrm{ha}^{-1}\right)$, carfentrazone $\left(25 \mathrm{~g} \mathrm{ha}^{-1}\right)$, chlorimuron-ethyl $\left(10 \mathrm{~g} \mathrm{ha}^{-1}\right)$, clomazone $(1,080$ $\left.\mathrm{g} \mathrm{ha}^{-1}\right)$, flumioxazin $\left(83 \mathrm{~g} \mathrm{ha}^{-1}\right)$, metribuzin $\left(480 \mathrm{~g} \mathrm{ha}^{-1}\right)$ metsulfuron-methyl $\left(2.4 \mathrm{~g} \mathrm{ha}^{-1}\right)$, glyphosate + carfentrazone $\left(720+25 \mathrm{~g} \mathrm{ha}^{-1}\right)$, glyphosate + chlorimuron-ethyl $\left(720+10 \mathrm{~g} \mathrm{ha}^{-1}\right)$, glyphosate + clomazone $\left(720+1080 \mathrm{~g} \mathrm{ha}^{-1}\right)$, glyphosate + flumioxazin $\left(720+83 \mathrm{~g} \mathrm{ha}^{-1}\right)$, glyphosate + metribuzin $\left(720+480 \mathrm{~g} \mathrm{ha}^{-1}\right)$, glyphosate + metsulfuron-methyl $\left(720+2.4 \mathrm{~g} \mathrm{ha}^{-1}\right)$, and an untreated control. Evaluations included percentage of phytotoxicity, plant height, root yield and starch content of cassava. The herbicides applied alone and in tank mix did not cause phytotoxicity in cassava plants after application. The herbicides applied alone and in tank mix did not reduce plant height, root yield and starch content of cassava compared to untreated control. The isolated application of residual herbicides and mixes with glyphosate were considered selective when applied after pruning of cassava plants 'Fécula Branca'.

Keywords: Manihot esculenta L. Crantz, tank mix, weeds

\section{Introdução}

A mandioca representa uma das mais importantes fontes de carboidratos da dieta humana em regiões tropicais e subtropicais (ElSharkawy, 2006; Jaramillo et al., 2005). No âmbito mundial, a mandioca destaca-se entre as principais culturas agrícolas, com produção acima de 251 milhões de toneladas por ano. $\mathrm{O}$ Brasil participa com 24,5 milhões desse total. Entre as tuberosas, perde apenas para a batata e encontra-se entre os seis principais produtos alimentares, representados pelo trigo, arroz, milho, batata, cevada e mandioca (CONAB, 2014).

O Estado do Paraná apresenta em torno de 161 mil hectares cultivados com a cultura da mandioca e uma produção estimada de 3,9 milhões de toneladas de raízes na safra 2013, com uma produtividade média de $23,9 \mathrm{t} \mathrm{ha}^{-1}$ (IBGE, 2014).

As duas principais categorias da mandioca são mandioca de mesa (aipim ou macaxeira) e mandioca para a indústria. Destacando-se que as variedades de mandioca destinadas para indústria de fécula apresentam ciclo entre 18 a 24 meses e frequentemente as plantas são podadas no período do repouso fisiológico ou dormência (10 a 12 meses após o plantio) (Takahashi, 1998; Sagrilo et al., 2002).

As plantas de mandioca têm seu crescimento e desenvolvimento afetado pela interferência de plantas daninhas, que, consequentemente, reduzem a produtividade e viabilidade econômica da cultura (Silva et al. 2009; Curcelli et al. 2010), e na qual a redução da produtividade foi relatada entre 89,8 a $100 \%$ (Peressin et al., 1998; Alabi et al., 2001; Chikoye et al., 2001; Johanns \& Contiero, 2006; Albuquerque et al.; 2008).

Desta forma, a partir da poda da mandioca inicia-se um novo período de desenvolvimento da cultura e a interferência das plantas daninhas pode prejudicar significativamente a produção e a qualidade das raízes, sendo necessária a adoção de medidas de controle.

Em grandes extensões de cultivos, a capina manual e mecânica apresentam alguns inconvenientes principalmente devido à escassez da mão de obra destinada às capinas, aos danos às raízes da cultura causados pelos cultivadores, além do baixo rendimento no controle. Estes métodos estão sendo pouco utilizados em áreas de lavouras comerciais, bem como podem apresentar custo de 22 a $64 \%$ superior em relação ao controle químico (Chikoye et al., 2006; Silva \& Chabaribery, 2006).

Desta maneira, fica evidente que o controle químico se apresenta como excelente alternativa para o manejo das plantas daninhas na cultura da mandioca. Contudo, no Brasil, 
apenas os herbicidas ametryn + clomazone, clethodim, clomazone, isoxaflutole e metribuzin são registrados para a cultura (Rodrigues \& Almeida, 2005).

Estes herbicidas apresentam maior ação de controle principalmente quando aplicados em pré-emergência das plantas daninhas. Porém, no período da poda das variedades de mandioca destinadas para a indústria, faz necessário a utilização de herbicidas pós-emergentes, uma vez que as plantas daninhas apresentam-se em diversos estádios de desenvolvimento.

Portanto, como as plantas de mandioca após o manejo da poda apresentam pouca capacidade de retenção das gotas de pulverização e de absorção dos herbicidas, torna-se possível conferir seletividade a herbicidas que apresentam amplo espectro de ação a exemplo do glyphosate. A mistura em tanque com pré-emergentes de diferentes mecanismos de ação pode contribuir na redução da pressão de seleção de biótipos resistentes e garantir maior eficiência no manejo das plantas daninhas, já que o glyphosate agiria no controle em pós-emergência e os herbicidas residuais agiriam no controle do banco de semente do solo.

Assim, objetivou-se avaliar a seletividade de herbicidas pré-emergentes e da mistura com glyphosate em aplicações após a poda da mandioca 'Fécula Branca'.

\section{Material e Métodos}

$\mathrm{O}$ experimento foi instalado na estação experimental do Instituto Agronômico do Paraná (IAPAR), no distrito de Porto Mendes, a $24^{\circ} 29^{\prime} 18^{\prime \prime}$ de latitude Sul e 54 18'11" de longitude Oeste e altitude aproximada de $218 \mathrm{~m}$, no município de Marechal Cândido Rondon/PR.

A temperatura média e as precipitações acumuladas mensalmente durante a condução do experimento são apresentadas na Figura 1.

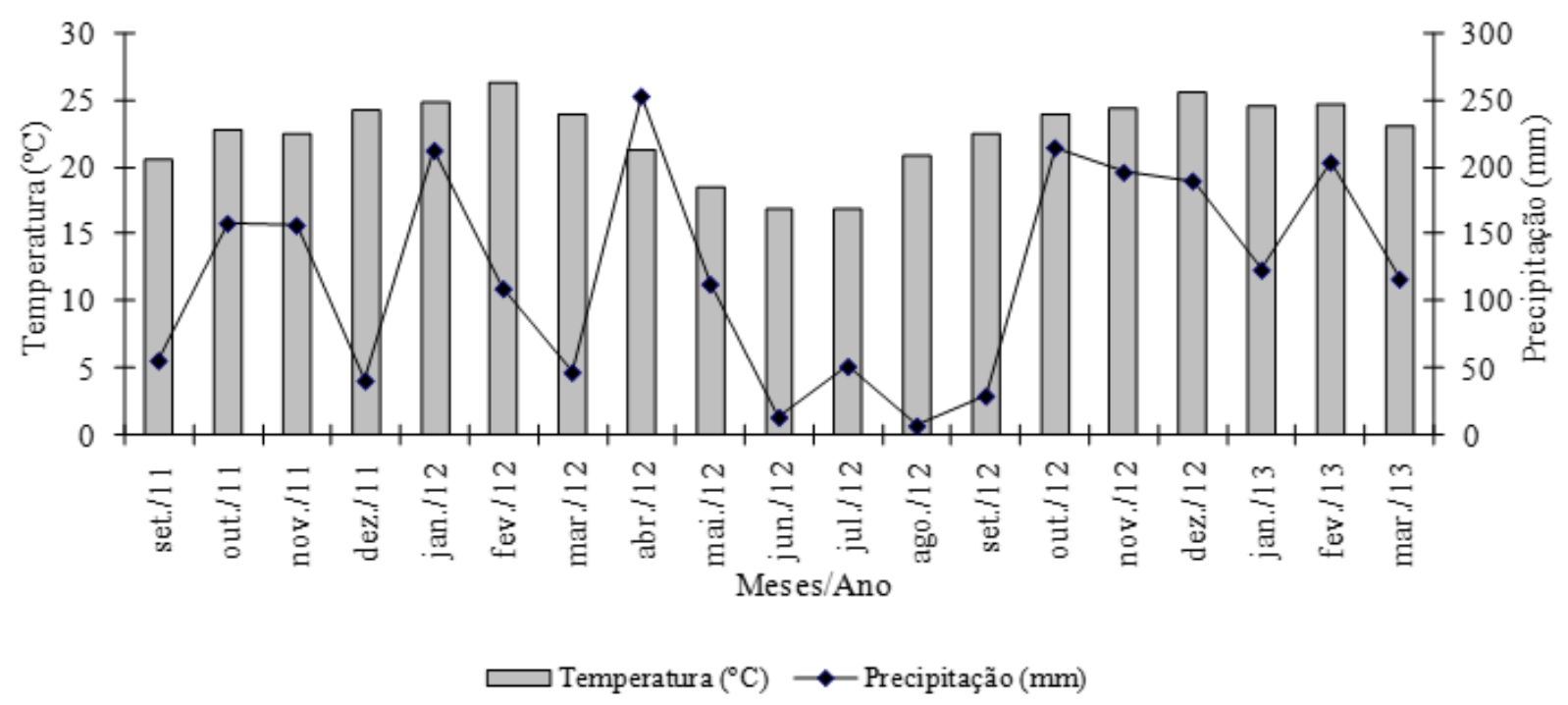

Figura 1. Temperatura média $\left({ }^{\circ} \mathrm{C}\right)$ e precipitação $(\mathrm{mm})$ durante o período de condução do experimento. Porto Mendes/PR, 2011-2013.

O plantio da variedade 'Fécula Branca' foi realizado em 15/09/2011 no sistema de plantio direto, com auxílio de plantadora mecanizada em linha no espaçamento de $0,9 \mathrm{x}$ $0,6 \mathrm{~m}$. No primeiro ciclo da cultura as plantas daninhas foram manejadas por meio do controle químico em pré-emergência com aplicação da mistura comercial ametryn + clomazone $(1.500$ $+1.000 \mathrm{~g} \mathrm{ha}^{-1}$ ).

A análise química do solo apresentou as seguintes características: $\mathrm{pH}\left(\mathrm{CaCl}_{2}\right)=5,6$; matéria orgânica $\left(\mathrm{g} \mathrm{dm}^{-3}\right)=22,6 ; \mathrm{P}\left(\mathrm{mg} \mathrm{dm}^{-3}\right)=$ 
4,0; H+Al, K, Ca, Mg, SB e CTC $\left(\mathrm{cmol}_{\mathrm{c}} \mathrm{dm}^{-3}\right)$ $=4,61 ; 0,51 ; 8,09 ; 1,88 ; 10,48$ e 15,09 ; respectivamente; e V $(\%)=69,45$; apresentando em sua composição textural $15,0 \%$ de areia; $30,0 \%$ de silte e $55,0 \%$ de argila. O solo foi classificado como Argissolo Vermelho eutrófico (PVe) (EMBRAPA, 1999).

$\mathrm{O}$ delineamento experimental utilizado foi o de blocos ao acaso, com quatro repetições. Os tratamentos avaliados foram: glyphosate (720 $\left.\mathrm{g} \mathrm{ha}^{-1}\right)$, carfentrazone (25 $\left.\mathrm{g} \mathrm{ha}^{-1}\right)$, chlorimuron-ethyl $\left(10 \mathrm{~g} \mathrm{ha}^{-1}\right)$, clomazone (1080 $\left.\mathrm{g} \mathrm{ha}^{-1}\right)$, flumioxazin $\left(83 \mathrm{~g} \mathrm{ha}^{-1}\right)$, metribuzin (480 $\left.\mathrm{g} \mathrm{ha}^{-1}\right)$ metsulfuron-methyl (2,4 $\left.\mathrm{g} \mathrm{ha}^{-1}\right)$, glyphosate + carfentrazone $\left(720+25 \mathrm{~g} \mathrm{ha}^{-1}\right)$, glyphosate + chlorimuron-ethyl $\left(720+10 \mathrm{~g} \mathrm{ha}^{-}\right.$ $\left.{ }^{1}\right)$, glyphosate + clomazone $\left(720+1080 \mathrm{~g} \mathrm{ha}^{-1}\right)$, glyphosate + flumioxazin $\left(720+83 \mathrm{~g} \mathrm{ha}^{-1}\right)$, glyphosate + metribuzin $\left(720+480 \mathrm{~g} \mathrm{ha}^{-1}\right)$, glyphosate + metsulfuron-methyl $(720+2,4 \mathrm{~g}$ $\left.\mathrm{ha}^{-1}\right)$, além de uma testemunha sem aplicação. Todas as parcelas foram mantidas livres da competição das plantas daninhas por meio de capinas manuais.

As parcelas foram constituídas de 4 linhas de mandioca espaçadas $0,9 \mathrm{~m}$ e com 0,6 $\mathrm{m}$ de comprimento. As aplicações dos tratamentos foram realizadas no final do primeiro ciclo de cultivo da mandioca 'Fécula Branca' após o manejo da poda das manivas 404 dias após o plantio (DAP) em 24/10/2012. A aplicação dos herbicidas foi realizada em 25/10/2012, um dia após a poda do caule.

Foi utilizado um pulverizador costal, pressurizado a $\mathrm{CO}_{2}$ e munido de barra com quatro pontas de jato plano (modelo Jacto 110LD-02), espaçadas $50 \mathrm{~cm}$ entre si e posicionada a $50 \mathrm{~cm}$ do alvo (caule). O consumo de calda foi equivalente a $200 \mathrm{~L} \mathrm{ha}^{-1}$, utilizando-se a pressão de $310 \mathrm{KPa}$. As aplicações foram realizadas no final da tarde, com condições de temperatura, umidade relativa do ar, velocidade do vento de $31,8^{\circ} \mathrm{C}, 53 \%, 3,96 \mathrm{~km} \mathrm{~h}^{-1}$, respectivamente.

As avaliações de fitointoxicação nas plantas de mandioca foram realizadas de forma visual, utilizando-se uma escala de percentual de notas, em que 0 (zero) corresponde a nenhuma injúria demonstrada pela planta de mandioca e 100 (cem) à morte total das plantas de mandioca (SBCPD, 1995). Os critérios usados para o estabelecimento das notas foram: inibição do crescimento, quantidade e uniformidade das injúrias, capacidade de rebrota das plantas e quantidade de plantas mortas. As avaliações foram realizadas aos 15, 20, 32 e 47 dias após a aplicação dos herbicidas (DAA).

Aos 47 DAA avaliou-se a altura (m) das plantas com régua graduada em centímetros, amostrando-se quatro plantas ao acaso nas duas linhas centrais das parcelas. Por ocasião da colheita das raízes da mandioca 556 DAP coletaram-se as plantas das duas linhas centrais das parcelas, desconsiderando uma planta de cada extremidade das linhas. Após a pesagem das raízes, determinou-se a produtividade e os dados foram extrapolados para $\mathrm{tha}^{-1} \mathrm{e}$ o teor de fécula (\%) foi determinado utilizando-se $o$ método da balança hidrostática conforme proposto por Grossman \& Freitas (1950).

Os resultados obtidos foram submetidos à análise de variância pelo teste $\mathrm{F}$, e as médias foram comparadas pelo teste Tukey a $5 \%$ de probabilidade.

\section{Resultados e Discussão}

As plantas de mandioca não apresentaram sintomas de fitointoxicação aos $15,20,32$ e 47 dias após a aplicação dos herbicidas (DAA). Os resultados observados nas demais variáveis avaliadas após a aplicação de herbicidas revelam que não houve diferenças entre os herbicidas e a testemunha sem aplicação (Tabela 1). Sendo que para os dados de altura de planta, produtividade de raízes e o teor de fécula da mandioca 'Fécula Branca', os valores mínimo e máximo obtidos foram 57,5 e $71,9 \mathrm{~cm} ; 41,4$ e $61,6 \mathrm{t} \mathrm{ha}^{-1}$ e 21,0 e $23,5 \%$, respectivamente.

A exemplo do solo argiloso utilizado no presente estudo, estes resultados corroboram com os obtidos por Scariot et al. (2013), que 
verificaram que o flumioxazin $\left(60 \mathrm{~g} \mathrm{ha}^{-1}\right)$ e a mistura do clomazone + flumioxazin $(900+50$ $\mathrm{g} \mathrm{ha}^{-1}$ ) foram seletivos às plantas de mandioca 'Cascuda', quando aplicados em préemergência em solo argiloso. Entretanto, Oliveira Jr. et al. (2001a), citaram que o flumioxazin (42 $\mathrm{g} \mathrm{ha}^{-1}$ ) aplicado em solo arenoso na pré-emergência da mandioca 'Espeto', provocou sintomas leves de fitointoxicação aos 28 dias após o plantio, porém houve redução de $27,6 \%$ na produção de raízes, quando comparado com a testemunha capinada.

Tabela 1. Altura de planta, produtividade de raízes e teor de fécula da mandioca 'Fécula Branca' após a aplicação de herbicidas. Porto Mendes/PR, 2011-2013.

\begin{tabular}{lcccc}
\hline \multicolumn{1}{c}{ Tratamento } & $\begin{array}{c}\text { Dose } \\
\left(\mathrm{g} \mathrm{ha}^{-1}\right)\end{array}$ & $\begin{array}{c}\text { Altura }^{1} \\
(\mathrm{~cm})\end{array}$ & $\begin{array}{c}\text { Produção de Raízes } \\
\left(\mathrm{t} \mathrm{ha}^{-1}\right)\end{array}$ & $\begin{array}{c}\text { Fécula } \\
(\%)\end{array}$ \\
\hline Testemunha sem aplicação & - & 61,6 & 57,3 & 22,4 \\
Glyphosate & 720 & 59,8 & 52,7 & 23,5 \\
Metsulfuron-methyl & 2,4 & 58,3 & 44,0 & 22,3 \\
Chlorimuron-ethyl & 10 & 58,1 & 41,4 & 23,3 \\
Flumioxazin & 83 & 59,8 & 48,1 & 22,5 \\
Metribuzin & 480 & 71,9 & 46,1 & 22,6 \\
Carfentrazone & 25 & 64,6 & 52,2 & 23,1 \\
Clomazone & 1.080 & 63,4 & 53,4 & 23,3 \\
Glyphosate + metsulfuron-methyl & $720+2,4$ & 67,2 & 48,6 & 23,0 \\
Glyphosate + chlorimuron-ethyl & $720+10$ & 60,3 & 48,5 & 23,1 \\
Glyphosate + flumioxazin & $720+83$ & 68,9 & 52,6 & 22,3 \\
Glyphosate + metribuzin & $720+480$ & 58,8 & 51,0 & 21,1 \\
Glyphosate + carfentrazone & $720+25$ & 57,5 & 61,6 & 21,5 \\
Glyphosate + clomazone & $720+1.080$ & 68,2 & 51,8 & 21,0 \\
\hline FTratamentos & & $1,285^{\mathrm{ns}}$ & $0,299^{\mathrm{ns}}$ & $1,596^{\mathrm{ns}}$ \\
FBlocos & & $19,940^{\mathrm{ns}}$ & $9,586^{* *}$ & $1,098^{\mathrm{ns}}$ \\
CV(\%) & & 13,17 & 37,46 & 5,68 \\
D.M.S. & & 20,9 & 47,9 & 3,2 \\
\hline n & & & \\
\hline
\end{tabular}

${ }^{\mathrm{n}}$ não significativo pelo teste ' $\mathrm{F}$ ' $(\mathrm{p}>0,05) ;{ }^{* *}$ significativo pelo teste ' $\mathrm{F}$ ' $(\mathrm{p}<0,01) ;{ }^{1}$ avaliação realizada aos 47 DAA.

Estes resultados podem ser justificados pelo fato de flumioxazin apresentar baixo valor de $\mathrm{K}_{\text {ow }}(2,55)$ (Rodrigues \& Almeida, 2005), o que corresponde na prática a maior solubilidade em períodos de maior precipitação, como maior mobilidade e menor adsorção da molécula em solos arenosos, que tendem a apresentar menor teor de matéria orgânica do que os solos argilosos (Oliveira et al., 1998; Ohmes \& Mueller, 2007; Szmigielski et al., 2009). Desta maneira, em solos argilosos como os do presente estudo, pode ocorrer menor absorção deste herbicida pela planta de mandioca sem causar fitointoxicação com consequências negativas na produtividade da cultura, ou pode haver tolerância diferencial entre as variedades de mandioca aos herbicidas.

Oliveira Jr. et al. (2001b), verificaram que o metribuzin (490 $\mathrm{g} \mathrm{ha}^{-1}$ ), não afetou negativamente o crescimento e a produtividade das cultivares de mandioca 'Espeto', 'Mico', 'Fécula Branca', 'IAC-14' e 'Fibra' em comparação com as respectivas testemunhas sem aplicação. Biffe et al. (2010), citaram que o metribuzin (360 e $720 \mathrm{~g} \mathrm{ha}^{-1}$ ) e sua mistura de metribuzin + isoxaflutole $\left(360+60 \mathrm{~g} \mathrm{ha}^{-1}\right)$, mostraram-se seletivos para os cultivares 'Fécula Branca' e 'Fibra', podendo ser usados 
como ferramentas no controle de plantas daninhas na cultura da mandioca.

Destaca-se que a seleção de outros herbicidas seletivos a mandioca, além do clomazone e do metribuzin que já são registrados para a cultura da mandioca, e que apresentam diferentes mecanismos de ação a exemplo do glyphosate, metsulfuron-methyl, chlorimuron-ethyl, flumioxazin e carfentrazone e das misturas em tanque com o glyphosate pode contribuir no manejo das plantas daninhas e evitar a seleção de biótipos resistentes aos herbicidas em áreas de cultivo de mandioca (Beckie \& Reboud, 2009; Vencill et al., 2012).

Monquero et al. (2001) constataram controle mais eficiente (97 a 100\%) de $C$. benghalensis, Galinsoga parviflora e Ipomoea grandifolia, quando utilizada a mistura de glyphosate $\left(420 \mathrm{~g} \quad \mathrm{ha}^{-1}\right)$ com herbicidas carfentrazone (30 $\left.\mathrm{g} \mathrm{ha}^{-1}\right)$, chlorimuron-ethyl (150 $\left.\mathrm{g} \mathrm{ha}^{-1}\right)$, flumioxazin $\left(50 \mathrm{~g} \mathrm{ha}^{-1}\right)$ e sulfentrazone $\left(70 \mathrm{~g} \mathrm{ha}^{-1}\right)$ do que o glyphosate isolado $\left(420 \mathrm{~g} \mathrm{ha}^{-1}\right)$, que apresentou controle das espécies de 77, 96 e 94\%, respectivamente.

Do mesmo modo, Maciel et al. (2011) citam que a mistura de glyphosate + chlorimuron-ethyl $\left(360+10 \mathrm{~g} \mathrm{ha}^{-1}\right)$ e glyphosate + carfentrazone $\left(360+4 \mathrm{~g} \mathrm{ha}^{-1}\right)$ apresentaram, aos 40 DAA, controle mais eficiente das plantas daninhas tolerantes ao glyphosate Commelina benghalensis (93,4 e 88,0\%, respectivamente) e Tridax procumbens $(92,5$ e $90,6 \%$, respectivamente) do que a aplicação isolada do glyphosate $\left(720 \mathrm{~g} \mathrm{ha}^{-1}\right)$ que apresentou controle de 64,4 e $73,0 \%$ para as referidas espécies, respectivamente.

Portanto, os resultados do presente trabalho indicam que a utilização do glyphosate (720 $\left.\mathrm{g} \mathrm{ha}^{-1}\right)$, carfentrazone $\left(25 \mathrm{~g} \mathrm{ha}^{-1}\right)$, chlorimuron-ethyl (10 $\left.\mathrm{g} \mathrm{ha}^{-1}\right)$, clomazone (1.080 $\left.\mathrm{g} \mathrm{ha}^{-1}\right)$, flumioxazin $\left(83 \mathrm{~g} \mathrm{ha}^{-1}\right)$, metribuzin (480 $\left.\mathrm{g} \mathrm{ha}^{-1}\right)$ metsulfuron-methyl $\left(2,4 \mathrm{~g} \mathrm{ha}^{-1}\right)$ e das misturas com o glyphosate pode ser uma excelente alternativa para o manejo das plantas daninhas em aplicações após a poda da mandioca 'Fécula Branca'. Bem como, ressalta- se o potencial de utilização das misturas em tanque para se explorar o amplo espectro de ação do glyphosate e o efeito residual dos herbicidas pré-emergentes sobre o banco de semente das plantas daninhas no solo.

\section{Conclusões}

Com base nos resultados obtidos, podese concluir que os herbicidas pré-emergentes e as suas respectivas misturas com glyphosate apresentaram-se seletivas, quando aplicados após a poda das plantas de mandioca 'Fécula Branca'.

\section{Agradecimentos}

Os autores agradecem ao Instituto Agronômico do Paraná (IAPAR) e a Associação Técnica das Indústrias de Mandioca do Paraná (ATMOP) pelo apoio técnico durante a realização do experimento.

\section{Referências}

ALABI, B.S. et al. Giant sensitive plant interference in cassava. Weed Science, v.49, n.2, p.171-176, 2001.

ALBUQUERQUE, J.A.A. et al. Interferência de plantas daninhas sobre a produtividade da mandioca (Manihot esculenta). Planta Daninha, v.26, n.2, p.279-289, 2008.

BECKIE, H.J.; REBOUD, X. Selecting for weed resistance: herbicide rotation and mixture. Weed Technology, v.23, n.3, p.363-370, 2009.

BIFFE, D.F. et al. Avaliação de herbicidas para dois cultivares de mandioca. Planta Daninha, v.28, n.4, p.807-816, 2010.

CHIKOYE, D. et al. Cogongrass suppression by intercropping cover crops in corn/cassava systems. Weed Science, v.49, n.5, p.658-667, 2001.

CHIKOYE, D. et al. Options for cogongrass (Imperata cylindrica) control in white guinea yam (Dioscorea rotundata) and cassava 
(Manihot esculenta). Weed Technology, v.20, n.3, p.784-792, 2006.

CONAB - COMPANHIA NACIONAL DE ABASTECIMENTO.

Mandioca-Raiz,

Farinha e Fécula. março de 2013, safra 2012/2013. Disponível em: <http://www.conab.gov.br/OlalaCMS/uploads/ arquivos/13_03_13_14_03_50_mandioca04a08 0313.pdf>. Acesso em: 26 de fevereiro de 2014.

CURCELLI, F. et al. Produção de cepa de duas variedades de mandioca em diferentes tratamentos de herbicidas. Revista Raízes e Amidos Tropicais, v.6, n.1, p.162-172, 2010.

EL-SHARKAWY, M.A. International research on cassava photosynthesis, productivity, eco physiology, and responses to environmental stresses in the tropics. Photosynthetica, v.44, n.4, p.481-512, 2006.

EMPRESA BRASILEIRA DE PESQUISA AGROPECUÁRIA - EMBRAPA. Serviço Nacional de Levantamento e Conservação de Solos. Sistema Brasileiro de Classificação de Solos. Rio de Janeiro: 1999. 412p.

GROSSMAN, J.; FREITAS, A.C. Determinação do teor de matéria seca pelo peso específico em raízes de mandioca. Revista Agronômica, v.14, n.160/162, p.75-80, 1950.

IBGE - INSTITUTO BRASILEIRO DE GEOGRAFIA E ESTATÍSTICA. Levantamento sistemático da produção agrícola: pesquisa mensal de previsão e acompanhamento das safras agrícolas no ano civil. Rio de Janeiro, v.27 n.2 p.1-84. Fevereiro/2014. Disponível em: <ftp://ftp.ibge.gov.br/Producao_Agricola/Leva ntamento_Sistematico_da_Producao_Agricola _[mensal]/Fasciculo/lspa_201402.pdf>. Acesso em: 16 de abril de 2014.

JARAMILLO, G. et al. Diallel analysis in cassava adapted to the mid altitude valleys environment. Crop Science, v.45, n.3, p.10581063, 2005.
JOHANNS, O.; CONTIERO, R.L. Efeitos de diferentes períodos de controle e convivência de plantas daninhas com a cultura da mandioca. Revista Ciência Agronômica, v.37, n.3, p.326331, 2006.

MACIEL, C.D.G. et al. Misturas em tanque com glyphosate para o controle de trapoeraba, ervade-touro e capim-carrapicho em soja $\mathrm{RR}^{\circledR}$. Revista Ceres, v.58, n.1, p.35-42, 2011.

MONQUERO P.A. et al. Glyphosate em mistura com herbicidas alternativos para o manejo de plantas daninhas. Planta Daninha, v.19, n.3, p.375-380, 2001.

OHMES, G.A.; MUELLER, T.C. Sulfentrazone adsorption and mobility in surface soil of the southern United States. Weed Technology, v.21, n.3, p.796-800, 2007.

OLIVEIRA JR., R.S. et al. Manejo químico de plantas daninhas em área de plantio direto de mandioca. Revista Brasileira de Herbicidas, v.2, n.3, p.99-106, 2001a.

OLIVEIRA JR., R.S. et al. Tolerância de cinco cultivares de mandioca (Manihot esculenta) a herbicidas. Planta Daninha, v.19, n.1, p.119125, 2001b.

OLIVEIRA, M.F. et al. Atividade dos herbicidas flumioxazin e metribuzin em diferentes solos. Planta Daninha, v.16, n.1, p.37-43, 1998.

PERESSIN, V.A. et al. Acúmulo de matéria seca na presença e na ausência de plantas infestantes no cultivar de mandioca SRT59 Branca de Santa Catarina. Bragantia, v.57, n.1, p.135-148, 1998.

RODRIGUES, B.N.; ALMEIDA, F.S. Guia de herbicidas. 5. ed. Londrina: Autores, 2005, 591p.

SAGRILO, E. et al. Efeito da época de colheita no crescimento vegetativo, na produtividade e na qualidade de raízes de três cultivares de mandioca. Bragantia, v.61, n.2, p.115-125, 2002. 
SBCPD - SOCIEDADE BRASILEIRA DA CIÊNCIA DAS PLANTAS DANINHAS. Procedimentos para instalação, avaliação e análise de experimentos com herbicidas. Londrina: $1995.42 \mathrm{p}$.

SCARIOT, C.A. et al. Seletividade e eficiência de herbicidas aplicados em pré-emergência na cultura da mandioca. Pesquisa Agropecuária Tropical, v.43, n.3, p.300-307, 2013.

SILVA, F.M.L. et al. Moléculas de herbicidas seletivos à cultura da mandioca. Revista Trópica: Ciências Agrárias e Biológicas, v.3, n.2, p.61-72, 2009.

SILVA, J.R.; CHABARIBERY, D. Coeficientes técnicos e custo de produção da mandioca para mesa na região de Mogi-Mirim, Estado de São Paulo. Informações Econômicas, v.36, n.1, p.26-32, 2006.

SZMIGIELSKI, A.M. et al. Development of a laboratory bioassay and effect of soil properties on sulfentrazone phytotoxicity in soil. Weed Technology, v.23, n.3, p.486-491, 2009.

TAKAHASHI, M. Épocas de poda na cultura da mandioca na região noroeste do Paraná, Brasil.

Brazilian Archives of Biology and Technology, v.41, n.4, p.495-500, 1998.

VENCILL, W.K. et al. Herbicide resistance: toward an understanding of resistance development and the impact of herbicideresistant crops. Weed Science, v.60, n.esp., p.230, 2012. 